\title{
Erratum to: Individualization of microfibrillated celluloses from oil palm empty fruit bunch: comparative studies between acid hydrolysis and ammonium persulfate oxidation
}

\author{
Kar Yin Goh - Yern Chee Ching • Cheng Hock Chuah • \\ Luqman Chuah Abdullah · Nai-Shang Liou
}

Published online: 29 March 2016

(C) Springer Science+Business Media Dordrecht 2016

\section{Erratum to: Cellulose (2016) 23:379-390 DOI 10.1007/s10570-015-0812-y}

In the original publication of the article, the following errors occurred inadvertently.

The error occurred during the sample preparation. We have discovered that Mylar foil that was used to wrap the samples is responsible for the peak at $2 \theta=26^{\circ}$ that was previously reported in the original publication as a peak for cellulose.

The online version of the original article can be found under doi:10.1007/s10570-015-0812-y.

K. Y. Goh · Y. C. Ching ( $₫)$

Department of Mechanical Engineering, Faculty of Engineering, University of Malaya, 50603 Kuala Lumpur, Malaysia

e-mail: chingyc@um.edu.my

K. Y. Goh · C. H. Chuah

Department of Chemistry, Faculty of Science, University of Malaya, 50603 Kuala Lumpur, Malaysia

L. C. Abdullah

Department of Chemical Engineering, Faculty of Engineering, University Putra Malaysia, 43400 Serdang, Malaysia

\section{N.-S. Liou}

Department of Mechanical Engineering, Southern Taiwan University of Science and Technology, Yungkang Dist., Tainan City 710, Taiwan, ROC
To conform with the required crystallographic conventions for cellulose, Eq. (1) is defined as follows:

$\mathrm{CrI}(\%)=\frac{I_{200}-I_{\mathrm{AM}}}{I_{200}} \times 100$

where CrI expresses crystallinity index, $I_{200}$ is the maximum intensity of diffraction at $2 \theta=22.4^{\circ}$ and $I_{\mathrm{AM}}$ is the intensity for the amorphous phase. This is the intensity of diffraction at $2 \theta=18^{\circ}$. The authors had mistakenly defined $I_{\mathrm{AM}}$ as stated in Eq. (1) as the minimum value, i.e., the intensity of the smaller peak at $15^{\circ}$, which the authors now understand as a legitimate crystalline peak, not a measure of the amorphous intensity.

Since it included the peak from Mylar, Fig. 4 in the original publication was incorrect and the corrected figure is provided in this Erratum. Figure 4 displays the experimental and simulated diffraction patterns of cellulose. Both raw fibers and APS-derived MFCs exhibit typical X-ray diffraction patterns of cellulose I. Based on the corrected diffraction patterns, the crystallinity index values for raw OPEFB and APS-oxidized MFCs were calculated to be 31 and $64 \%$, respectively. This error also misled us to postulate that there was transformation from cellulose I to cellulose II. Hence, in the part of "Results and discussion" (in section "Crystallinity studies"), paragraph 5 "Cellulose can be generally classified into ........, which is cellulose II" shall be deleted. However, a partial 


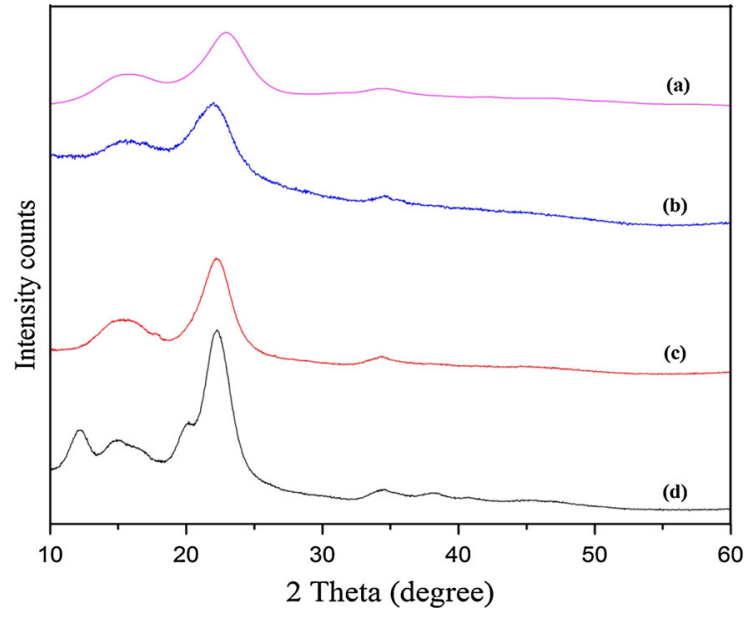

Fig. 4 Powder diffraction pattern calculated based on $a$ cellulose I structure $\left(\mathrm{pwhm}=3.8^{\circ}\right)$ using mercury software and the experimental powder diffraction patterns of $b$ raw OPEFB, $c$ APS-oxidized MFCs and $d$ sulfuric acid-hydrolyzed MFCs transformation to cellulose II is seen for the sulfuric acid-hydrolyzed MFCs. This is probably due to the treatment $17.5 \% \mathrm{NaOH}$ to remove hemicellulose. 